\title{
Simple Adaptive Oblivious Transfer without Random Oracle
}

\author{
Kaoru Kurosawa and Ryo Nojima \\ 1 Ibaraki University, Japan \\ 2 NICT, Japan
}

\begin{abstract}
Adaptive oblivious transfer (OT) is a two-party protocol which simulates an ideal world such that the sender sends $M_{1}, \cdots, M_{n}$ to the trusted third party (TTP), and the receiver receives $M_{\sigma_{i}}$ from TTP adaptively for $i=1,2, \cdots k$. This paper shows the first pairing-free fully simulatable adaptive OT. It is also the first fully simulatable scheme which does not rely on dynamic assumptions. Indeed our scheme holds under the DDH assumption.
\end{abstract}

Keywords: Adaptive OT, Fully Simulatable, DDH, Standard Model.

\section{Introduction}

In a non-adaptive $(k, n)$ oblivious transfer (OT) scheme which is denoted by $O T_{k}^{n}$ 6114, a sender has $n$ secret strings $M_{1}, \cdots, M_{n}$, and a receiver has $k$ secret choice indices $\sigma_{1}, \cdots, \sigma_{k} \in\{1, \cdots, n\}$. At the end of the protocol, the receiver learns $M_{\sigma_{1}}, \cdots, M_{\sigma_{k}}$ (only), and the sender learns nothing on $\sigma_{1}, \cdots, \sigma_{k}$. Efficient OT schemes are important because $O T_{1}^{4}$ is a key building block for secure multi-party computation 20712.

In an adaptive $(k, n)$ oblivious transfer protocol which is denoted by $O T_{k \times 1}^{n}$, the receiver chooses $\sigma_{i}$ adaptively depending on $M_{\sigma_{1}}, \cdots, M_{\sigma_{i-1}}$ [15. In other words, $O T_{k \times 1}^{n}$ is a two-party protocol $(S, R)$ which simulates an ideal world protocol $\left(S^{\prime}, R^{\prime}\right)$ such that

1. the sender $S^{\prime}$ sends $M_{1}, \cdots, M_{n}$ to the trusted third party (TTP), and

2. the receiver $R^{\prime}$ receives $M_{\sigma_{i}}$ from TTP adaptively for $i=1,2, \cdots k$, where the receiver chooses $\sigma_{i}$ based on $M_{\sigma_{1}}, \cdots, M_{\sigma_{i-1}}$.

Adaptive OT has wide applications such as oblivious database searches, secure multiparty computation and etc, too.

As a security notion of OT (for both non-adaptive and adaptive), half simulatability was considered until recently 15161118 . This definition requires

- (Sender's privacy.) For any receiver $R$ in the real world, there exists a receiver $\hat{R}$ in the ideal world such that the outputs of $R$ and $\hat{R}$ are indistinguishable.

- (Receiver's privacy.) For any input to the receiver, the view of the sender must be indistinguishable. (Note that the honest sender outputs nothing.) 
However, Naor and Pinkas noticed that there can be a practical attack on a half simulatable adaptive OT [15].

To solve this problem, Camenisch, Neven and shelat formalized a notion of full simulatability [2]. In this definition, we consider a pair of outputs of the sender and the receiver. Although the honest sender outputs nothing, a malicious sender may output its view in the execution of the protocol. Full simulatability now requires that

- (Sender's privacy) For any receiver $\hat{R}$ in the real world, there exists a receiver $\hat{R}^{\prime}$ in the ideal world such that $\left(S_{\text {out }}^{\prime}, \hat{R}_{\text {out }}^{\prime}\right)$ is indistinguishable from $\left(S_{\text {out }}, \hat{R}_{\text {out }}\right)$, where $A_{\text {out }}$ denotes the output of $A$.

- (Receiver's privacy) For any sender $\hat{S}$ in the real world, there exists a sender $\hat{S}^{\prime}$ in the ideal world such that $\left(\hat{S}_{\text {out }}^{\prime}, R_{\text {out }}^{\prime}\right)$ is indistinguishable from $\left(\hat{S}_{\text {out }}^{\prime}\right.$, $\left.R_{\text {out }}\right)$.

They then showed a fully simulatable adaptive OT in the random oracle model, and one in the standard model, respectively [2].

We focus on the standard model in this paper 1 Then all fully simulatable adaptive OT known so far have been constructed based on pairing, and they rely on dynamic assumptions such as $q$-strong DH assumption. For example, Camenisch et al.'s $O T_{k \times 1}^{n}$ relies on $q$-strong DH assumption and $q$-PDDH assumption. Green and Hohenberger's $O T_{k \times 1}^{n}$ relies on $q$-hidden LRSW assumption 9]. (This scheme achieves UC security.) Jarecki and Liu's $O T_{k \times 1}^{n}$ relies on the decisional $q$-DHI assumption [10].

This paper shows the first pairing-free fully simulatable adaptive OT. It is also the first fully simulatable scheme which does not rely on dynamic assumptions. Indeed our scheme holds under the DDH assumption. While the previous schemes use a signature scheme as a building block 2 our scheme utilizes ElGamal encryption scheme. (Hence we do not need a pairing.)

Our scheme is conceptually very simple and efficient. The initialization phase and each transfer phase are constant round protocols. Thus the total round complexity is proportional to $k$.

Finally we extend our scheme to a fully simulatable non-adaptive OT which requires constant rounds. Green and Hohenberger showed a fully simulatable non-adaptive $\mathrm{OT}_{k}^{n}$ based on pairing under the decisional BDH assumption [8]. On the other hand, our $\mathrm{OT}_{k}^{n}$ is pairing-free and relies on the DDH assumption.

Lindell showed a fully simulatable $O T_{1}^{2}$ under DDH, Paillier's decisional $N$ th residuosity, and quadratic residuosity assumptions as well as under the assumption that homomorphic encryption exists [13. (He claimed that they can be extended to $O T_{k}^{n}$.) Under the DDH assumption, our $O T_{1}^{2}$ is more efficient than the Lindell's scheme [13.

\footnotetext{
${ }^{1}$ In the random oracle model, Ogata and Kurosawa showed an adaptive OT based on Chaum's blind signature scheme [18. Camenisch, Neven and shelat 2 proved that it is fully simulatable as well as they corrected a flaw of 18. Green and Hohenberger showed a scheme under the decisional BDH assumption [8].

${ }^{2}$ Maybe because an adaptive OT shown by Ogata and Kurosawa [18] utilizes Chaum's blind signature scheme.
} 
Table 1. Fully simulatable Adaptive OT without RO

\begin{tabular}{|c|c|c|c|}
\hline scheme & pairing & dynamic assumption & assumption \\
\hline Camenisch et al. [2] & yes & yes & $q$-strong DH and $q$-PDDH \\
\hline Green and Hohenberger [9] & yes & yes & $q$-hidden LRSW (UC secure) \\
\hline Jarecki and Liu [10] & yes & yes & $q$-DHI \\
\hline Proposed & no & no & DDH \\
\hline
\end{tabular}

\section{Preliminaries}

\subsection{Notations}

In this paper, we denote a security parameter by $\tau \in \mathbb{N}$. All the algorithms take $\tau$ as the first input and run in (expected) polynomial-time in $\tau$. We denote probabilistic polynomial-time by PPT for short. We often do not write the security parameter explicitly.

\subsection{Proof Systems}

To design our scheme, we use several proof systems. We follow the definitions described in 452 .

Let $R=\{(\alpha, \beta)\} \subseteq\{0,1\}^{*} \times\{0,1\}^{*}$ be a binary relation $R$ such that $|\beta| \leq$ $\operatorname{poly}(\alpha)$ for all $(\alpha, \beta) \in R$, where poly is some polynomial. We only consider the relation $R$ such that $(\alpha, \beta) \in R$ can be decided in polynomial in $|\alpha|$ for all $(\alpha, \beta)$. We define $L_{R}=\{\alpha \mid \exists \beta$ such that $(\alpha, \beta) \in R\}$.

Proof of Membership (PoM): A pair of interacting algorithms (P, V), called a prover and a verifier, is a proof of membership (PoM) for a relation $R$ if the completeness and soundness are satisfied. Here, we say that $(\mathrm{P}, \mathrm{V})$ satisfies the completeness if for all $(\alpha, \beta) \in R$, the probability of $\mathrm{V}(\alpha)$ accepting a conversation with $\mathrm{P}(\alpha, \beta)$ is 1 . Also we say that $(\mathrm{P}, \mathrm{V})$ satisfies the soundness if for all $\alpha \notin L_{R}$ and all $\mathrm{P}^{*}(\alpha)$ (including cheating provers), the probability of $\mathrm{V}(\alpha)$ accepting the conversation with $\mathrm{P}^{*}$ is negligible in $|\alpha|$. We say that this probability as soundness error of the proof system.

Proof of Knowledge (PoK): We say a pair of interacting algorithms (P, V) is PoK for a relation $R$ with knowledge error $\kappa \in[0,1]$ if it satisfies completeness described above and has an expected polynomial-time algorithm, called knowledge extractor, E. Here, the algorithm $\mathrm{E}$ is a knowledge extractor for a relation $R$ if possibly cheating $\widehat{P}$ has probability $\epsilon$ of convincing $\mathrm{V}$ to accept $\alpha$, then $\mathrm{E}$, when given black-box access to $\widehat{P}$, outputs a witness $\beta$ for $\alpha$ with probability $\epsilon-\kappa$.

Witness Indistinguishability (WI): A proof system $(\mathrm{P}, \mathrm{V})$ is perfect WI if for every $\left(\alpha, \beta_{1}\right),\left(\alpha, \beta_{2}\right) \in R$, and any PPT cheating verifier, the output of $\widehat{\mathrm{V}}(\alpha)$ (including cheating verifier) after interacting with $\mathrm{P}\left(\beta_{1}\right)$ and that of $\widehat{\mathrm{V}}(\alpha)$ after interacting with $\mathrm{P}\left(\beta_{2}\right)$ are identically distributed. 
Zero Knowledge (ZK): We say that a proof system $(\mathrm{P}, \mathrm{V})$ is perfect $\mathrm{ZK}$ if there exists an expected polynomial-time algorithm Sim, called a simulator, such that for any PPT cheating verifier $\widehat{V}$ and any $(\alpha, \beta) \in R$, the outputs of $\widehat{V}(\alpha)$ after interacting with $\mathrm{P}(\beta)$ and that of $\operatorname{Sim}^{\widehat{V}(\alpha)}(\alpha)$ are identically distributed.

\section{$3 \quad k$-Out-of- $n$ Oblivious Transfer}

In this section, we present a UC-like definition of fully simulatable non-adaptive OT. Similarly, we present a UC-like definition of fully simulatable adaptive OT.

We consider a weak model of UC framework as follows.

- At the beginning of the game, an adversary A can corrupt either a sender $\mathrm{S}$ or a receiver $\mathrm{R}$, but not both.

- A can send a message (which will be denoted by $A_{\text {out }}$ ) to an environment $\mathcal{Z}$ after the end of the protocol. (A cannot communicate with $\mathcal{Z}$ during the protocol execution.)

The ideal functionalities of $\mathrm{OT}_{k}^{n}$ and $\mathrm{OT}_{k \times 1}^{n}$ will be shown below. For a protcol $\pi=(\mathrm{S}, \mathrm{R})$, define $\operatorname{Adv}(\mathcal{Z})$ as

$$
\operatorname{Adv}(\mathcal{Z})=\mid \operatorname{Pr}(\mathcal{Z}=1 \text { in the real world })-\operatorname{Pr}(\mathcal{Z}=1 \text { in the ideal world }) \mid
$$

\subsection{Non-adaptive $k$-Out-of- $n$ Oblivious Transfer}

In the ideal world of $\mathrm{OT}_{k}^{n}$, the ideal functionality $\mathcal{F}_{\text {non }}$, an ideal world adversary $\mathrm{A}^{\prime}$ and an environment $\mathcal{Z}$ behave as follows.

(Initialization phase:)

1. An environment $\mathcal{Z}$ sends $\left(M_{1}, \cdots, M_{n}\right)$ to the dummy sender $\mathrm{S}^{\prime}$.

2. $\mathrm{S}^{\prime}$ sends $\left(M_{1}^{*}, \cdots, M_{n}^{*}\right)$ to $\mathcal{F}_{\text {non }}$, where $\left(M_{1}^{*}, \cdots, M_{n}^{*}\right)=\left(M_{1}, \cdots, M_{n}\right)$ if $\mathrm{S}^{\prime}$ is not corrupted.

(Transfer phase:)

1. $\mathcal{Z}$ sends $\left(\sigma_{1}, \cdots, \sigma_{k}\right)$ to the dummy receiver $\mathrm{R}^{\prime}$, where $1 \leq \sigma_{i} \leq n$.

2. $\mathrm{R}^{\prime}$ sends $\left(\sigma_{1}^{*}, \cdots, \sigma_{k}^{*}\right)$ to $\mathcal{F}_{\text {non }}$, where $\left(\sigma_{1}^{*}, \cdots, \sigma_{k}^{*}\right)=\left(\sigma_{1}, \cdots, \sigma_{k}\right)$ if $\mathbf{R}^{\prime}$ is not corrupted.

3. $\mathcal{F}_{\text {non }}$ sends received to an ideal process adversary $\mathrm{A}^{\prime}$.

4. $\mathrm{A}^{\prime}$ sends $b=1$ or 0 to $\mathcal{F}_{\text {non }}$, where $b=1$ if $\mathrm{S}^{\prime}$ is not corrupted.

5. $\mathcal{F}_{\text {non }}$ sends $Y$ to $\mathrm{R}^{\prime}$, where

$$
Y=\left\{\begin{array}{cl}
\left(M_{\sigma_{1}}^{*}, \cdots, M_{\sigma_{k}}^{*}\right) & \text { if } b=1 \\
\perp & \text { if } b=0
\end{array}\right.
$$

6. $\mathrm{R}^{\prime}$ sends $Y$ to $\mathcal{Z}$.

After the end of the protocol, $\mathrm{A}^{\prime}$ sends a message $A_{\text {out }}^{\prime}$ to $\mathcal{Z}$. Finally $\mathcal{Z}$ outputs 1 or 0 . 
In the real world, a protocol $(\mathrm{S}, \mathrm{R})$ is executed without $\mathcal{F}_{\text {non }}$, where the environment $\mathcal{Z}$ and a real world adversary $A$ behave in the same way as above.

Definition 1. We say that $(\mathrm{S}, \mathrm{R})$ is secure against the sender (receiver) corruption if for any real world adversary $\mathrm{A}$ who corrupts the sender $\mathrm{S}$ (the receiver $\mathrm{R})$, there exists an ideal world adversary $\mathrm{A}^{\prime}$ who corrupts the dummy sender $\mathrm{S}^{\prime}$ (the dummy receiver $\mathrm{R}^{\prime}$ ) such that for any environment $\mathcal{Z}, \operatorname{Adv}(\mathcal{Z})$ is negligible.

Definition 2. We say that $(\mathrm{S}, \mathrm{R})$ is a fully simulatable $O T_{k}^{n}$ if it is secure against the sender corruption and the receiver corruption.

\subsection{Adaptive $k$-Out-of- $n$ Oblivious Transfer}

In the ideal world of $\mathrm{OT}_{k \times 1}^{n}$, the ideal functionality $\mathcal{F}_{\text {adapt }}$, an ideal world adversary $A^{\prime}$ and an environment $\mathcal{Z}$ behave as follows.

(Initialization phase:)

1. An environment $\mathcal{Z}$ sends $\left(M_{1}, \cdots, M_{n}\right)$ to the dummy sender $\mathrm{S}^{\prime}$.

2. $\mathrm{S}^{\prime}$ sends $\left(M_{1}^{*}, \cdots, M_{n}^{*}\right)$ to $\mathcal{F}_{\text {adapt }}$, where $\left(M_{1}^{*}, \cdots, M_{n}^{*}\right)=\left(M_{1}, \cdots, M_{n}\right)$ if $\mathrm{S}^{\prime}$ is not corrupted.

(Transfer phase:) For $i=1, \cdots, k$,

1. $\mathcal{Z}$ sends $\sigma_{i}$ to the dummy receiver $\mathrm{R}^{\prime}$, where $1 \leq \sigma_{i} \leq n$.

2. $\mathrm{R}^{\prime}$ sends $\sigma_{i}^{*}$ to $\mathcal{F}_{\text {adapt }}$, where $\sigma_{i}^{*}=\sigma_{i}$ if $\mathrm{R}^{\prime}$ is not corrupted.

3. $\mathcal{F}_{\text {adapt }}$ sends received to an ideal process adversary $A^{\prime}$.

4. $\mathrm{A}^{\prime}$ sends $b=1$ or 0 to $\mathcal{F}_{\text {adapt }}$, where $b=1$ if $\mathrm{S}^{\prime}$ is not corrupted.

5. $\mathcal{F}_{\text {adapt }}$ sends $Y_{i}$ to $\mathrm{R}^{\prime}$, where

$$
Y_{i}=\left\{\begin{array}{c}
M_{\sigma_{i}}^{*} \text { if } b=1 \\
\perp \text { if } b=0
\end{array}\right.
$$

6. $\mathrm{R}^{\prime}$ sends $Y_{i}$ to $\mathcal{Z}$.

After the end of the protocol, $\mathrm{A}^{\prime}$ sends a message $A_{\text {out }}^{\prime}$ to $\mathcal{Z}$. Finally $\mathcal{Z}$ outputs 1 or 0 .

In the real world, a protocol $(\mathrm{S}, \mathrm{R})$ is executed without $\mathcal{F}_{\text {adapt }}$, where the environment $\mathcal{Z}$ and a real world adversary $A$ behave in the same way as above.

Definition 3. We say that $(\mathrm{S}, \mathrm{R})$ is secure against the sender (receiver) corruption if for any real world adversary $\mathrm{A}$ who corrupts the sender $\mathrm{S}$ (the receiver $\mathrm{R})$, there exists an ideal world adversary $\mathrm{A}^{\prime}$ who corrupts the dummy sender $\mathrm{S}^{\prime}$ (the dummy receiver $\mathrm{R}^{\prime}$ ) such that for any environment $\mathcal{Z}, \operatorname{Adv}(\mathcal{Z})$ is negligible.

Definition 4. We say that $(\mathrm{S}, \mathrm{R})$ is a fully simulatable $O T_{k \times 1}^{n}$ if it is secure against the sender corruption and the receiver corruption. 


\subsection{Remarks}

Our definition of fully simulatable adaptive OT is weaker than the UC security because our adversaries A cannot communicate with $\mathcal{Z}$ during the protocol execution. On the other hand, it is stronger than that of 2 which is not UC-like. In our definition, $\mathcal{Z}$ chooses $\sigma_{i}$. Hence $\sigma_{i}$ can depend on all of $\left(M_{1}, \cdots, M_{n}\right)$. In the definition of [2], receiver chooses $\sigma_{i}$. Hence $\sigma_{i}$ can depend on $\left(M_{\sigma_{1}}, \cdots, M_{\sigma_{i-1}}\right)$ only.

\section{Our Fully Simulatable Adaptive OT}

In this section, we show an adaptive $\mathrm{OT}_{k \times 1}^{n}$ based on ElGamal encryption scheme, and prove its full simulatability under the DDH assumption.

Let $\mathbb{G}$ be a multiplicative group of prime order $q$. Then the DDH assumption states that, for every PPT distinguisher D,

$$
\epsilon_{\mathrm{DDH}}(\mathrm{D})=\left|\operatorname{Pr}\left(\mathrm{D}\left(g, g^{\alpha}, g^{\beta}, g^{\alpha \beta}\right)=1\right)-\operatorname{Pr}\left(\mathrm{D}\left(g, g^{\alpha}, g^{\beta}, g^{\gamma}\right)=1\right)\right|
$$

is negligible, where the probability is taken over the random bits of $D$, the random choice of the generator $g$, and the random choice of $\alpha, \beta, \gamma \in \mathbb{Z}_{q}$. We denote

$$
\epsilon_{\mathrm{DDH}}=\max \left\{\epsilon_{\mathrm{DDH}}(\mathrm{D})\right\},
$$

where the maximum is taken over all PPT distinguishers D.

The initialization phase and each transfer phase are constant round protocols. Hence the total round complexity is proportional to $k$.

\section{Initialization Phase}

1. The sender chooses $\mathbb{G}, g$ and $\left(x_{1}, \cdots, x_{n}, r\right) \in\left(\mathbb{Z}_{q}\right)^{n+1}$ randomly, and computes $h=g^{r}$.

2. For $i=1, \cdots, n$, the sender computes

$$
C_{i}=\left(A_{i}, B_{i}\right)=\left(g^{x_{i}}, M_{i} \cdot h^{x_{i}}\right),
$$

where $M_{1}, \cdots, M_{n} \in \mathbb{G}$.

3. The sender sends $\left(\mathbb{G}, h, C_{1}, \cdots, C_{n}\right)$.

4. The sender proves by ZK-PoK that he knows $r$.

The protocol stops if the receiver rejects.

\section{The $j$ th Transfer Phase}

1. The receiver chooses a choice index $1 \leq \sigma_{j} \leq n$ based on $M_{\sigma_{1}}, \cdots, M_{\sigma_{j-1}}$.

2. The receiver chooses $u \in \mathbb{Z}_{q}$ randomly and computes $U=\left(A_{\sigma_{j}}\right)^{u}$.

He then sends $U$.

3. The receiver proves in WI-PoK that he knows $u$ such that

$$
U=A_{1}^{u} \vee \cdots \vee U=A_{n}^{u} .
$$

The protocol stops if the sender rejects. 
4. The sender computes $V=U^{r}$ and sends $V$.

5. The sender proves that $(g, h, U, V)$ in ZK-PoM that it is a DDH-tuple. The protocol stops if the receiver rejects.

6. The receiver obtains $M_{\sigma_{j}}$ by computing $B_{\sigma_{j}} / V^{1 / u}$.

Three ZK or WI proof systems in the scheme are constructed efficiently as follows.

- An efficient 4-round ZK-PoK exists which can be used in the initialization phase. It is obtained by applying the technique of [4] to Schnorr's identification scheme [19.

- An efficient 3-round WI-PoK exists which can be used in the transfer phase. It is implemented by applying the or-composition technique [5] to [19].

- An efficient 4-round ZK-PoM exists which can be used in the transfer phase. It comes from the confirmation protocol of Chaum's undeniable signature scheme (which is a ZK-PoM for the DDH-tuple [3]).

Theorem 1. The above protocol is a fully-simulatable adaptive $O T_{k \times 1}^{n}$ under the DDH assumption.

The proof is given in Section 6 .

\section{Extension to Fully Simulatable Non-adaptive OT}

In this section, we extend our adaptive OT to a fully simulatable non-adaptive OT which requires constant rounds.

\subsection{How to Prove Many DDH-Tuples}

We show a 4-round ZK-PoM which proves that $\left(g, h, U_{1}, V_{1}\right), \cdots,\left(g, h, U_{k}, V_{k}\right)$ are all DDH-tuples.

1. The receiver sends random $\left(a_{1}, \cdots, a_{k}\right)$.

2. The sender proves that $\left(g, h, \prod_{i=1}^{k} U_{i}^{a_{i}}, \prod_{i=1}^{k} V_{i}^{a_{i}}\right)$ is a DDH-tuple by using the confirmation protocol of [3].

The confirmation protocol of [3] is a 4-round ZK-PoM on a DDH-tuple. Hence the above protocol runs in 4-round. (Step 1 and the 1st round of the confirmation protocol are merged.)

Lemma 1. Suppose that some $\left(g, h, U_{i}, V_{i}\right)$ is not a DDH-tuples. Then $\left(g, h, \prod_{i=1}^{k} U_{i}^{a_{i}}, \prod_{i=1}^{k} V_{i}^{a_{i}}\right)$ is a DDH-tuples with negligible probability.

Proof. Assume that $U_{i}=g^{x_{i}}$ and $V_{i}=h^{y_{i}}$ for $i=1, \cdots, k$. Then

$$
\begin{aligned}
& \prod_{i=1}^{k} U_{i}^{a_{i}}=g^{\sum_{i=1}^{k} a_{i} x_{i}} \\
& \prod_{i=1}^{k} V_{i}^{a_{i}}=h^{\sum_{i=1}^{k} a_{i} y_{i}}
\end{aligned}
$$


Suppose that $\left(g, h, U_{1}, V_{1}\right)$ is not a DDH-tuples. That is, $x_{1} \neq y_{1}$. Then for any values of $a_{2}, \cdots, a_{k}$, there exists a unique $a_{1}$ such that

$$
\sum_{i=1}^{k} a_{i}\left(x_{i}-y_{i}\right)=0 \bmod q .
$$

Hence the numbers of $\left(a_{1}, \cdots, a_{k}\right)$ which satisfies eq. (11) is equal to $q^{k-1}$. Therefore

$$
\operatorname{Pr} \text { (eq. (11) holds) }=q^{k-1} / q^{k}=1 / q \text {. }
$$

This means that $\left(g, h, \prod_{i=1}^{k} U_{i}^{a_{i}}, \prod_{i=1}^{k} V_{i}^{a_{i}}\right)$ is a DDH-tuples with negligible probability.

Theorem 2. The above protocol is a ZK-PoM on many DDH-tuples.

Proof. The completeness is clear. The zero-knowledgeness follows from that of the confirmation protocol of 3 . The soundness follows from Lemma 1 and that of the confirmation protocol of [3].

\subsection{Constant Round $\mathrm{OT}_{k}^{n}$}

In this section, we modify our $\mathrm{OT}_{k \times 1}^{n}$ to obtain a constant round $\mathrm{OT}_{k}^{n}$ as follows.

- At step 4 of the initialization phase, the sender sends $\left(\mathbb{G}, h, A_{1}, \cdots, A_{n}\right)$.

- At the end of the transfer phase, the sender sends $\left(B_{1}, \cdots, B_{n}\right)$.

- In the transfer phase, run step 3 in parallel (still it is a WI protocol). At step 5, the sender proves that $\left(g, h, U_{1}, V_{1}\right), \cdots,\left(g, h, U_{k}, V_{k}\right)$ are all DDHtuples by using the ZK-PoM of Sec 5.1.

Theorem 3. The proposed $O T_{k}^{n}$ is a constant round fully-simulatable $\mathrm{OT}_{k}^{n}$ under the DDH assumption.

The proof is similar to that of Theorem 1 .

\section{Proof of Theorem 1}

We first prove that the proposed scheme is secure against sender corruption. We next prove that it is secure against receiver corruption.

\subsection{Security against Sender Corruption}

Lemma 2. The proposed scheme is secure against sender corruption.

Proof. For every real-world adversary A who corrupts the sender, we construct an ideal-world adversary $A^{\prime} \operatorname{such}$ that $\operatorname{Adv}(\mathcal{Z})$ is negligible.

We will consider a sequence of games Game 0 , Game $1, \cdots$, Game $_{4}$, where Game $_{0}$ is the real world experiment of Sec 3 and and $\mathrm{Game}_{4}$ is the ideal world experiment, respectively. Let

$$
\operatorname{Pr}\left(\mathrm{GAME}_{i}\right)=\operatorname{Pr}\left(\mathcal{Z}=1 \text { in } \text { Game }_{i}\right) .
$$


Game $_{0}$ : This is the real world experiment such that the sender is controlled by an adversary $A$. Hence

$$
\operatorname{Pr}\left(\mathrm{GAME}_{0}\right)=\operatorname{Pr}(\mathcal{Z}=1 \text { in the real world }) .
$$

Game $_{1}$ : This is the same as the previous game except for the following. In the initialization phase, if the receiver accepts the ZK-PoK, then he extracts $r$ from $A$ by running the knowledge extractor $E_{1}$ which is allowed to rewind $A$. This game outputs $\perp$ if the extractor $\mathrm{E}_{1}$ fails in extracting $r$. Unless this happens, these two games are identical. Therefore,

$$
\left|\operatorname{Pr}\left(\mathrm{GAME}_{0}\right)-\operatorname{Pr}\left(\mathrm{GAME}_{1}\right)\right| \leq \kappa_{1},
$$

where $\kappa_{1}$ be the knowledge error of the extractor.

Game $_{2}$ : This is the same as the previous game except for the following. In each transfer phase, if the receiver accepts the ZK-PoM which proves that $(g, h, U, V)$ is a DDH-tuple, then he obtains $M_{\sigma_{i}}$ by computing $B_{\sigma_{i}} / A_{\sigma_{i}}^{r}$. These two games are identical unless the above $M_{\sigma_{i}}$ is different from $B_{\sigma_{j}} / V^{1 / u}$. This happens if the receiver accepts the ZK-PoM even though $(g, h, U, V)$ is not a DDH-tuple. Hence

$$
\left|\operatorname{Pr}\left(\mathrm{GAME}_{1}\right)-\operatorname{Pr}\left(\mathrm{GAME}_{2}\right)\right| \leq k \kappa_{3},
$$

where $\kappa_{3}$ is the soundness error probability of ZK-PoM.

Game $_{3}$ : This is the same as the previous game except for the following. In each transfer phase, the receiver computes $U$ as $U=A_{1}^{u}$. (The receiver can still obtain $M_{\sigma_{i}}$ as can be seen from $\mathrm{Game}_{2}$.) Since our WI-PoK is perfect,

$$
\operatorname{Pr}\left(\mathrm{GAME}_{2}\right)=\operatorname{Pr}\left(\mathrm{GAME}_{3}\right) .
$$

Game $_{4}$ : This game is the ideal world experiment in which an ideal-world adversary $A^{\prime}$ plays the role of the receiver of $G_{a m e}$ and uses $A$ as a blackbox. $A^{\prime}$ can do this because the receiver does not use $\sigma_{1}, \cdots, \sigma_{k}$ in $\mathrm{Game}_{3}$.

Finally $A^{\prime}$ outputs what $A$ outputs. It is easy to see that $\mathrm{Game}_{3}$ and $\mathrm{Game}_{4}$ are identical from a view point of $\mathcal{Z}$. Hence

$$
\operatorname{Pr}\left(\mathrm{GAME}_{3}\right)=\operatorname{Pr}\left(\mathrm{GAME}_{4}\right) .
$$

Further

$$
\operatorname{Pr}\left(\mathrm{GAME}_{4}\right)=\operatorname{Pr}(\mathcal{Z}=1 \text { in the ideal world }) .
$$

Now, we can summarize this lemma as follows:

$$
\begin{aligned}
\operatorname{Adv}(\mathcal{Z}) & =\left|\operatorname{Pr}\left(\mathrm{GAME}_{4}\right)-\operatorname{Pr}\left(\mathrm{GAME}_{0}\right)\right| \\
& \leq \sum_{i=0}^{3}\left|\operatorname{Pr}\left(\mathrm{GAME}_{i+1}\right)-\operatorname{Pr}\left(\mathrm{GAME}_{i}\right)\right| \\
& \leq \kappa_{1}+k \kappa_{3} .
\end{aligned}
$$




\subsection{Security against Receiver Corruption}

Lemma 3. The proposed scheme is secure against receiver corruption under the DDH assumption.

Proof. For every real-world adversary A who corrupts the receiver, we construct an ideal-world adversary $A^{\prime}$ such that $\operatorname{Adv}(\mathcal{Z})$ is negligible.

We will consider a sequence of games $\mathrm{Game}_{0}, \mathrm{Game}_{1}, \cdots, \mathrm{Game}_{5}$, where Game is the real world experiment of Sec 3 , and Game ${ }_{5}$ is the ideal world experiment.

Game $_{0}$ : This is the real world experiment such that the receiver is controlled by an adversary A. Hence

$$
\operatorname{Pr}\left(\mathrm{GAME}_{0}\right)=\operatorname{Pr}(\mathcal{Z}=1 \text { in the real world }) .
$$

$G_{a m e}$ : This is the same as the previous game except for the following. In each transfer phase, instead of running the ZK-PoM which proves that $(g, h, U, V)$ is a DDH-tuple, the sender runs the zero-knowledge simulator of the ZK-PoM which is allowed to rewind A. Since the ZK-PoM is perfect ZK, we have

$$
\operatorname{Pr}\left(\mathrm{GAME}_{1}\right)=\operatorname{Pr}\left(\mathrm{GAME}_{0}\right) .
$$

Game $_{2}$ : This is the same as the previous game except for the following. In each transfer phase, if the sender accepts the WI-PoK, then she extracts $u$ from A by running the knowledge extractor $E_{2}$ which is allowed to rewind $A$. This game outputs $\perp$ if the extractor $E_{2}$ fails in extracting $u$. Unless this happens, these two games are identical. Therefore,

$$
\left|\operatorname{Pr}\left(\mathrm{GAME}_{2}\right)-\operatorname{Pr}\left(\mathrm{GAME}_{1}\right)\right| \leq k \kappa_{2},
$$

where $\kappa_{2}$ is the knowledge error of the extractor.

$\mathrm{Game}_{3}$ : This is the same as the previous game except for that the sender computes $V$ as $V=\left(B_{\sigma} / M_{\sigma}\right)^{u}$ instead of $V=U^{r}$. It is clear that there is no essential difference between two games. Therefore,

$$
\operatorname{Pr}\left(\mathrm{GAME}_{3}\right)=\operatorname{Pr}\left(\mathrm{GAME}_{2}\right) .
$$

Game $_{4}$ : This is the same as the previous game except for that the sender uses a random $M_{i}^{\prime}$ to compute each $C_{i}$ in the initialization phase. The difference $\left|\operatorname{Pr}\left(\mathrm{GAME}_{4}\right)-\operatorname{Pr}\left(\mathrm{GAME}_{3}\right)\right|$ is still negligible by the semantic security of the ElGamal cryptosystem which is implied by the DDH assumption.

Claim. If the DDH problem is hard then $\left|\operatorname{Pr}\left(\mathrm{GAME}_{4}\right)-\operatorname{Pr}\left(\mathrm{GAME}_{3}\right)\right|$ is negligible. More concretely,

$$
\left|\operatorname{Pr}\left(\mathrm{GAME}_{4}\right)-\operatorname{Pr}\left(\mathrm{GAME}_{3}\right)\right| \leq \epsilon_{\mathrm{DDH}} .
$$

The proof of this claim is given later. 
Game $_{5}$ : This game is the ideal world experiment in which an ideal-world adversary $A^{\prime}$ plays the role of the sender of Game $e_{4}$, and uses $A$ as a blackbox. $A^{\prime}$ can do this because the sender does not use $M_{1}, \cdots, M_{n}$ in $\mathrm{Game}_{4}$.

Finally $A^{\prime}$ outputs what $A$ outputs. It is easy to see that Game ${ }_{4}$ and Game $\mathbf{F}_{5}$ are identical from a view point of $\mathcal{Z}$. Hence

$$
\operatorname{Pr}\left(\mathrm{GAME}_{4}\right)=\operatorname{Pr}\left(\mathrm{GAME}_{5}\right) .
$$

Further

$$
\operatorname{Pr}\left(\mathrm{GAME}_{5}\right)=\operatorname{Pr}(\mathcal{Z}=1 \text { in the ideal world }) .
$$

Now, we can summarize this lemma as follows:

$$
\begin{aligned}
\operatorname{Adv}(\mathcal{Z}) & =\left|\operatorname{Pr}\left(\operatorname{GAME}_{5}\right)-\operatorname{Pr}\left(\operatorname{GAME}_{0}\right)\right| \\
& \leq \sum_{i=0}^{4}\left|\operatorname{Pr}\left(\operatorname{GAME}_{i+1}\right)-\operatorname{Pr}\left(\mathrm{GAME}_{i}\right)\right| \\
& \leq k \kappa_{2}+\epsilon_{\mathrm{DDH}} .
\end{aligned}
$$

To complete the proof, we must provide the proof of the claim. To do so, we need the following lemma ${ }^{3}$ which can be thought of as an "extended" version of the DDH assumption.

Lemma 4 (Lemma 4.2 in [17]). If there exists a probabilistic algorithm D with running time $t$ such that

$$
\begin{aligned}
& \mid \operatorname{Pr}\left(\mathrm{D}\left(g, g^{r}, g^{x_{1}}, \cdots, g^{x_{n}}, g^{r x_{1}}, \cdots, g^{r x_{n}}\right)=1\right) \\
& \quad-\operatorname{Pr}\left(\mathrm{D}\left(g, g^{r}, g^{x_{1}}, \cdots, g^{x_{n}}, g^{z_{1}}, \cdots, g^{z_{n}}\right)=1\right) \mid \geq \epsilon
\end{aligned}
$$

where the probability is taken over the random bits of $\mathrm{D}$, the random choice of the generator $g$ in $\mathbb{G}$, and the random choice of $x_{1}, \cdots, x_{n}, r, z_{1}, \cdots, z_{n} \in \mathbb{Z}_{q}$, then there exists a probabilistic algorithm with running time $n \cdot \operatorname{poly}(\tau)+t$ that breaks the $D D H$ assumption with probability $\geq \epsilon$ with some polynomial poly.

We now show a proof of the claim.

Proof (of the claim). Let Game ${ }_{3}^{\prime}\left(\mathrm{Game}_{4}^{\prime}\right)$ be the same as Game 3 (Game 4 ) except for the following. In the initialization phase, instead of running the ZK-PoK in which the sender proves that he knows $r$, the sender runs the zero-knowledge simulator of the ZK-PoK which is allowed to rewind A. Since the ZK-PoK is perfect ZK, it holds that

$$
\begin{aligned}
& \operatorname{Pr}\left(\mathrm{GAME}_{3}^{\prime}\right)=\operatorname{Pr}\left(\mathrm{GAME}_{3}\right), \\
& \operatorname{Pr}\left(\mathrm{GAME}_{4}^{\prime}\right)=\operatorname{Pr}\left(\mathrm{GAME}_{4}\right) .
\end{aligned}
$$

${ }^{3}$ Naor and Reingold proved it by using the random reducibility of the DDH-tuple. 
We now construct a DDH distinguisher D in the sense of Lemma 4. The input to $\mathrm{D}$ is $\left(g, h, g^{x_{1}}, \cdots, g^{x_{n}}, y_{1}, \cdots, y_{n}\right)$, where $y_{i}=g^{r x_{i}}$ or $g^{z_{i}}$, Our D simulates $\mathcal{Z}$, A and the sender of $\mathrm{Game}_{3}^{\prime}$ or $\mathrm{Game}_{4}^{\prime}$ faithfully except for that in the initialization phase, D simulates the sender by using $\left(g, h, g^{x_{1}}, \cdots, g^{x_{n}}\right)$, and $h_{i}=y_{i}$ for each $i$. Finally D outputs 1 iff $\mathcal{Z}$ outputs 1 .

It is easy to see that D simulates Game ${ }_{3}^{\prime}$ if $y_{i}=g^{r x_{i}}$ for each $i$, and $\mathrm{Game}_{4}^{\prime}$ otherwise. Therefore

$$
\left|\operatorname{Pr}\left(\mathrm{GAME}_{4}^{\prime}\right)-\operatorname{Pr}\left(\mathrm{GAME}_{3}^{\prime}\right)\right| \leq \epsilon_{\mathrm{DDH}}
$$

Hence eq.(2) holds.

\section{$7 \quad$ Fully Simulatable $O T_{1}^{2}$}

We have constructed a fully-simulatable adaptive OT under the DDH assumption in the standard model. It is clear that we can obtain a fully-simulatable $(1,2)$-OT $\left(O T_{1}^{2}\right)$ as a special case.

On the other hand, Lindell showed a fully simulatable $O T_{1}^{2}$ under DDH, Paillier's decisional $N$ th residuosity, and quadratic residuosity assumptions as well as under the assumption that homomorphic encryption exists in the standard model 13 .

Let's compare our scheme with Lindell's $O T_{1}^{2}$ which is based on the DDH assumption. His scheme builds on the $O T_{1}^{2}$ of [16] and uses a cut-and-choose technique. The computational cost and the communication cost are $O(\ell)$ times larger than those of our first scheme to achieve

$$
\operatorname{Adv}(\mathcal{Z}) \leq 2^{-\ell+2}
$$

Hence our scheme is more efficient.

\section{References}

1. Brassard, G., Crépeau, C., Robert, J.M.: All-or-Nothing Disclosure of Secrets. In: Odlyzko, A.M. (ed.) CRYPTO 1986. LNCS, vol. 263, pp. 234-238. Springer, Heidelberg (1987)

2. Camenisch, J.L., Neven, G., Shelat, A.: Simulatable Adaptive Oblivious Transfer. In: Naor, M. (ed.) EUROCRYPT 2007. LNCS, vol. 4515, pp. 573-590. Springer, Heidelberg (2007)

3. Chaum, D.: Zero-Knowledge Undeniable Signatures. In: Damgård, I.B. (ed.) EUROCRYPT 1990. LNCS, vol. 473, pp. 458-464. Springer, Heidelberg (1991)

4. Cramer, R., Damgård, I., MacKenzie, P.D.: Efficient Zero-Knowledge Proofs of Knowledge Without Intractability Assumptions. In: Imai, H., Zheng, Y. (eds.) PKC 2000. LNCS, vol. 1751, pp. 354-373. Springer, Heidelberg (2000)

5. Cramer, R., Damgård, I.B., Schoenmakers, B.: Proofs of Partial Knowledge and Simplified Design of Witness Hiding Protocols. In: Desmedt, Y.G. (ed.) CRYPTO 1994. LNCS, vol. 839, pp. 174-187. Springer, Heidelberg (1994) 
6. Even, S., Goldreich, O., Lempel, A.: A Randomized Protocol for Signing Contracts. Commun. ACM 28(6), 637-647 (1985)

7. Goldreich, O., Micali, S., Wigderson, A.: How to Play any Mental Game or A Completeness Theorem for Protocols with Honest Majority. In: STOC 1987, pp. 218-229 (1987)

8. Green, M., Hohenberger, S.: Blind Identity-Based Encryption and Simulatable Oblivious Transfer. In: Kurosawa, K. (ed.) ASIACRYPT 2007. LNCS, vol. 4833, pp. 265-282. Springer, Heidelberg (2007)

9. Green, M., Hohenberger, S.: Universally Composable Adaptive Oblivious Transfer. In: Pieprzyk, J. (ed.) ASIACRYPT 2008. LNCS, vol. 5350, pp. 179-197. Springer, Heidelberg (2008)

10. Jarecki, S., Liu, X.: Efficient Oblivious Pseudorandom Function with Applications to Adaptive OT and Secure Computation of Set Intersection. In: Reingold, O. (ed.) TCC 2009. LNCS, vol. 5444, pp. 577-594. Springer, Heidelberg (2009)

11. Kalai, Y.T.: Smooth Projective Hashing and Two-Message Oblivious Transfer. In: Cramer, R. (ed.) EUROCRYPT 2005. LNCS, vol. 3494, pp. 78-95. Springer, Heidelberg (2005)

12. Kilian, J.: Founding Cryptography on Oblivious Transfer. In: STOC 1988, pp. 20-31 (1988)

13. Lindell, A.Y.: Efficient Fully-Simulatable Oblivious Transfer. In: Malkin, T.G. (ed.) CT-RSA 2008. LNCS, vol. 4964, pp. 52-70. Springer, Heidelberg (2008)

14. Naor, M., Pinkas, B.: Oblivious Transfer and Polynomial Evaluation. In: STOC 1999, pp. 245-254 (1999)

15. Naor, M., Pinkas, B.: Oblivious Transfer with Adaptive Queries. In: Wiener, M. (ed.) CRYPTO 1999. LNCS, vol. 1666, pp. 573-590. Springer, Heidelberg (1999)

16. Naor, M., Pinkas, B.: Efficient Oblivious Transfer Protocols. In: SODA 2001, pp. 448-457 (2001)

17. Naor, M., Reingold, O.: Number-Theoretic Constructions of Efficient Pseudorandom Functions. J. ACM 51(2), 231-262 (2004)

18. Ogata, W., Kurosawa, K.: Oblivious Keyword Search. J. Complexity 20(2-3), 356371 (2004); Cryptology ePrint Archive: Report 2002/182

19. Schnorr, C.-P.: Efficient Signature Generation by Smart Cards. J. Cryptology 4(3), 161-174 (1991)

20. Yao, A.C.-C.: How to Generate and Exchange Secrets. In: FOCS 1986, pp. 162-167 (1986) 